\title{
ASPECTOS DE ENFERMAGEM NA PREVENÇÃO PRIMÁRIA DAS DOENÇAS ISQUÊMICAS DO CORAÇÃO
}

\author{
Diná de Almeida Lopes Monteiro da Cruz*
}

CRUZ, D.A.L.M. da. Aspectos de enfermagem na prevençăo primária das doenças isquêmicas do coraçăo. Rev. Esc. Enf. USP, Săo Paulo, 22(2):215-221, ago. 1988.

Este artigo apresenta informaçðes sobre alguns fatores de risco para as doenças isquêmicas do coracão que auxiliam na fundamentaçảo das açoes preventivas do enfermeiro nessa área.

UNITERMOS: Cardiologia. Fatores de risco. Assistência de enfermagem.

\section{INTRODUÇÃO}

Análises dos dados de mortalidade têm apontado as doenças cárdiovasculares como problema de saúde importante para a nossa população sendo que as doenças isquêmicas do coração (DIC) são determinantes desses coeficientes (LAURENTI' ${ }^{13}$, LOLIO \& LAURENTI's).

Através de estudos epidemiológicos pode ser estabelecida a relação de certas caracteristicas pessoais, hábitos ou estilos de vida com a ocorrência das doenças coronarianas. Essas caracteristicas, chamadas fatores de risco, estão presentes com maior freqüência em individuos que desenvolvem DIC do que na população geral.

Nas DIC esses fatores de risco são múltiplos, complexos, interrelacionados e difíceis de serem isolados; entre os mais estudados incluem-se: idade, sexo, hipertensão arterial, fumo de cigarros, hipercolesterolemia, hiperglicemia, vida sedentária, obesidade, história familiar de doença cardíaca, tipo de personalidade e estresse. (HEYDEN', KANNEL ${ }^{9}$, WOLINSKY ${ }^{20}$ ).

Dentre estes, destacam-se os que são potencialmente reversiveis, direcionando assim os esforços para prevenção das DIC.

Em alguns paises desenvolvidos, tem-se observado um declínio nos coeficientes de mortalidade por DIC aventando-se a possibilidade de que estão sendo feitas as "coisas certas" para sua prevenção (STAMLER ${ }^{17}$ ), associando-se a essa tendência alteraçסes nos hábitos de vida como: redução do fumo, menor consumo de gorduras animais e colesterol, e possivelmente um controle mais efetivo da hipertensão e melhor tratamento da cardiopatia isquêmica (HEYDEN').

Conclui-se também, num estudo realizado no municipio de São Paulo, que a mortalidade por DIC apresentou tendência ascendente de 1.950 até 1.976 , havendo um declínio a partir dessa época (LOLIO \& LAURENTI ${ }^{15}$ ). Apesar de não haver ne-

- Enfermeira. Auxiliar de Ensino do Departamento de Enfermagem Medico-Cirúrgica da Escola de Enfermagem da USP - disciplina Enfermagem Médico-Cirúrgica. 
nhuma conclusão acerca das razóes do declínio observado, é possivel que exista alguma relação com modificação de fatores de risco e com os progressos no tratamento e controle das DIC.

Essas possiveis relaçðes indicam uma área importante para atuação do enfermeiro que junto aos seus clientes, pacientes e suas famílias provavelmente encontrará pessoas submetidas a fatores de risco que necessitam de assistência tendo em vista a prevenção das DIC.

A prevenção das doenças isquêmicas do coração envolvem a identificação dos fatores de risco a que as pessoas podem estar submetidas e a modificação daqueles passiveis de serem alterados ${ }^{3}$. Assim, é importante que o enfermeiro seja capaz de reconhecer os fatores de risco e esteja preparado para fazer as orientaçðes necessárias para a sua modificação.

Apesar das dificuldades relativas à mudança de comportamento, a pessoa que recebe assistência de enfermagem precisa, no minimo, de estar informada quanto aos riscos de desenvolver DIC impostos por hábitos ou comportamentos seus. Além de ter conhecimentos e habilidades referentes ao ensino do paciente/cliente, ao processo de ensino-aprendizagem como um todo, o enfermeiro deverá ter conhecimentos específicos acerca dos fatores de risco relacionados ao desenvolvimento das DIC.

A seguir, os principais fatores de risco das DIC serão abordados com a finalidade de contribuir para a fundamentação teórica necessária para cssa atuação do enfermeiro.

\section{HIPERTENSĀO ARTERIAL}

A hipertensão arterial é um dos fatores que concorrem para acelerar o processo de aterosclerose, independente de ser lábil ou não, sistólica ou diastólica, basal ou secundária, de estar relacionada a idade ou sexo (KANNEL"). Cabe salientar, porém, que muitas pessoas hipertensas podem ter outros fatores de risco associados, tais como: obesidade, hiperlipidemia, inatividade física $c$ estresse que aumentam o risco das DIC.

Apesar das dificuldades de se determinar níveis pressóricos normais é aceito que indivíduos com pressão diastólica acima de $90 \mathrm{~mm} \mathrm{Hg} \mathrm{e} \mathrm{acima} \mathrm{de} 80 \mathrm{~mm} \mathrm{Hg}$, para pessoas com idade inferior a 40 anos, sejam orientados para modificação de alguns hábitos (KAPLAN"'). A restrição de sódio e manutenção de peso são recomendaçôes prudentes, especialmente no caso de adultos jovens que têm hipertensão leve ou moderada e nenhum outro fator de risco cardiovascular (ALLRED \& AYERS').

Apesar das controvérsias acerca dos benefícios da restrição de ingestão de sódio para a prevenção e controle da hipertensão a sua redução até ao mínimo tolerado certamente não produzirá nenhum efeito deletério e pode trazer algum benefício (KAPLAN ${ }^{11}$ ).

O sal contém grande quantidade de sódio ( $2,3 \mathrm{mg} /$ colher de chá), porém outros alimentos são fontes importantes de sódio e devem ser cvitados, como por excmplo: enlatados, embutidos, molho de soja, azeitonas, pickles, carnes defumadas, atum $\mathrm{em}$ óleo (KERN \& GAWI.INK ${ }^{12}$ ).

Considerando-se a possibilidade de estarem associados outros fatores de risco, deve-se orientar também quanto a técnicas de relaxamento, desenvolvimento de atjvidade física regular e eliminação do hábito de fumar. 
A pessoa hipertensa deve ser encaminhada para avaliação médica, e o enfermeiro deve assisti-la na sua evoluçào que inclui a orientaçăo quanto a cronicidade e possiveis complicaçoes da hipertensåo, quanto a importância de controle periódico da pressão e aderência às orientaçoes e ao tratamento instituído.

\section{HIPERLIPIDEMIA}

O metabolismo lipídico, especialmente o do colesterol, desempenha papel importante nas doenças cardiovasculares, tanto isoladamente quanto associado a outros fatores (GOTTO \& WITTELS).

Apesar de não haver provas absolutas quanto a prevenção das DIC através da adequação dietética, há uma forte evidência quanto a sua efetividade e segurança. Essa adequação visa principalmente a diminuiçăo dos níveis de colesterol, que são afetados por diferentes componentes alimentares. Os ácidos graxos saturados e o colesterol da dieta tendem a aumentar o colesterol sérico, enquanto que'os ácidos graxos insaturados tendem a diminui-los (TURPEINEM ${ }^{19}$ ). Assim, a reduçăo da ingestão de ácidos graxos saturados é a primeira recomendaçãc. As principais fontes destes são as gorduras animais de bovinos, de suinos e de aves. Em segundo lugar indica-se substituir o conteúdo de ácidos graxos saturados da dieta por ácidos graxos polinsaturados, o que pode ser obtido pela substituição de gorduras animais, por óleos vegetais (milho, soja e girassol). Muitas gorduras de peixes são também boas fontes de ácidos graxos insaturados (TURPEINEM ${ }^{19}$ ).

O terceiro e não menos importante ponto a ser observado é a diminuição da ingestão de alimentos ricos em colesterol tais como a gema do ovo, figado e rins (TURPEINEM ${ }^{19}$ ). Conforme DEL NERO ${ }^{4}$, a parte mais importante do ovo é a clara que é rica em albumina, mas ainda continua bastante arraigado entre nós o uso da "gemada" por ainda ser popularmente considerada um bom "fortificante". Outros alimentos ricos em colesterol que devem ser eliminados da dieta são: manteiga, queijos preparados com leite integral, sorvetes, leite integral, iogurte com leite integral, oleo de oliva, banha, gordura de coco, bacon, carnes em conserva, lingüiças, salsi chas, carne de porco, carneiro, pele de frango, carne de pato e ganso, peixes conservados em oleo, camarão, abacate, chocolate, coco, molhos, etc. (KERN \& GAWLINK'2).

A influência de outros lipidios tem sido estudada, especialmente a das lipoproteinas. Ainda não há uma conclusão, mas parece que os niveis plasmáticos de $\mathrm{HDL}$ (lipoproteina de alta densidade) exercem ação protetora contra as DIC, e as mulheres têm niveis séricos médios de HDL mais altos que os homens (ALLRED \& AYERS'). As medidas para aumentar o HDL sérico ainda não são claras e comprovadamente seguras. No entanto, é importante alertar os indivíduos com $\mathrm{HDL}$ baixo para que adotem estilo de vida o mais saudável possivel, incluindo: redução de peso para obesos, exercicios regulares, parar de fumar e evitar o uso de contraceptivos orais (ALLRED \& AYERS').

\section{TABAGISMO}

O fumo de cigarros é um dos fatores de risco mais freqüentes das doenças insquêmicas do coração. A mortalidade por DIC é $70 \%$ maior em homens que fumam 
um maço de cigarros por dia que em não fumantes, e os que param de fumar, perdem progressivamente o risco adicional. As mulheres que associam o uso de contraceptivos orais e o fumo têm um aumento na mortalidade por cardiopatia isquêmica (ARONOW \& KAPLAN²).

Não se chegou a uma conclusão se o fumo favorece a aterosclerose ou se causa unicamente um efeito agudo por diminuir transitoriamente a oferta de oxigênio ao miocárdio e aumentar a sua demanda concomitantemente (HEYDEN ${ }^{7}$ ). O efeito agudo explicaria a redução do risco em individuos que deixam de fumar. Assim, a relação entre as DIC e o tabagismo permanece apenas parcialmente explicada.

As razoes preventivas dirigem-se para dois pontos: em primeiro lugar, quanto à aquisição do hábito, os adolescentes adquirem o hábito possivelmente por pressão dos amigos para que pareçam mais maduros, além do encorajamento adicional proveniente do exemplo dos pais e dos meios de comunicação. Muitos estão conscientes dos prejuízos a longo prazo causados pelo fumo quando começam a fumar e apesar de desejarem parar de fumar logo cedo, apenas $25 \%$ o consegue (ARONOW \& KAPLAN ${ }^{2}$ ). Assim, quando um adolescente começa a fumar a probabilidade de que mantenha o hábito por toda vida é muito grande. Em segundo lugar, quanto aos individuos que estão já habituados au fumo, é importante ressaltar que mesmo que o fumo seja iniciado por questoes psicossociais, os efeitos farmacológicos da nicotina podem causar dependência (ARONOW \& KAPLAN:).

Apesar das dificuldades referidas por quem tem o hábito, a recomendação básica é clara: evitar, diminuir ou parar de fumar (ARONOW \& KAPLAN²).

\section{DIABETES}

Não são claros os mecanismos pelos quais a hiperglicemia participa no processo da aterogênese, aumentando o risco de DIC entre os diabèticos e pessoas com tolerância anormal a glicose (ALLRED \& AYERS', HEYDEN', KAPLAN ${ }^{\prime \prime)}$ ). Sabe-se, no entanto, que os individuos diabéticos têm também, frequientemente, outros fatores de risco associados (ALLRED \& AYERS').

Como a prevenção das diabetes nào é possivel e há controvérsias se o controle da glicemia reduz o risco de DIC ou não (ALLRED \& AYERS'), as orientaçoes mais importantes referem-se ao controle dos outros fatores de risco associados e a necessidade de acompanhamento médico e de enfermagem quando houver alteraçoes de glicemia, que devem ser dectadas o mais precocemente possivel.

\section{OBESIDADE:}

Não há consistência nos dados quanto a contribuição independente da obesidade para as DIC. A obesidade geralmente é acompanhada por outros fatores de risco como hiperlipidemia, hipertensào arterial e intolerância a glicose, sendo que as alıeraçoes de peso, geralmente correspondem a alteraçoes nesses outros riscos cardiovasculares (KANNEL").

Independentemente desses questionamentos, a aquisição e manutenção de peso corporal adequado só podem trazer beneficios, sejam físicos, sociais ou psicológicos. A abordagem quanto a prevenção primária das DIC entre os obesos deve 
centrar-se nas modificaçōes dietéticas (restrição de calorias, colesterol e sódio, e substituição dos ácidos graxos saturados por insaturados) e na prática règular de exercício físico visando a redução de peso.

\section{VIDA SEDENTÁRIA}

Apesar das controvérsias existentes, algumas investigaçoes mostram a influência dos exercicios físicos sobre o nivel dos lipidios circulantes (GIANNINIs). De qualquer maneira, havendo ou não outros fatores de risco associados o esporte é necessário para que se evitem tensoes e se metabolize melhor o excesso de colesterol que ingerimos na alimentaçào diária (DEL NERO ${ }^{+}$).

Ao selecionar-se um modo apropriado de exercicio fisico, deve-se considerar o tipo, intensidade, duração e frequência do mesmo (LEON \& BLACKBURN ${ }^{1+}$ ). As atividades recomendadas para um adequado condicionamento cardiocirculatório são as aeróbicas, como natação, ciclismo e especialmente as caminhadas em terrenos planos. A manutenção da regularidade na prática esportiva é imprescindivel para o condicionamento físico. Contudo, é preciso ressaltar que o exercício físico nào é isento de riscos, sendo frequiente a ocorrência de morte súbita em pistas de corrida, quadras de tênis, etc. Por isso, o American College of Sports Medicine, citado por ALLRED \& AYERS' recomenda que seja feita avaliação clínica com teste de esforço antes de se inieiar qualquer programa para os seguintes individuos:

1. assintomáticos, fisicamente inativos, com 35 anos de idade ou mais e sem nenhum outro fator de risco coronariano;

2. assintomáticos, fisicamente ativos ou inativos, em qualquer idade, com qualquer risco coronariano;

3. assintomáticos ou sintomáticos, ativos ou inativos em qualquer idade, com história prévia de DIC;

4. submetidos a qualquer outra condição clinica que exija atenção.

\section{TIPO DE PERSONALIDADE E ESTRESSE}

As tensōes psicossociais relativas a situaçðes de vida ou circunstâncias culturais, têm sido relacionadas às DIC por condicioná-las ou agravá-las (KANNEL ${ }^{4}$ ). Os mecanismos pelos quais essas situaçoes estariam ligadas ao desenvolvimento prematuro das DIC não são conhecidos e, freqüentemente, outros padroes comportamentais de risco (fumo, tipo de alimentaçào, atividade física) estão associados ao estresse.

JEKINS $^{\star}$ subdivide os fatores psicossociais e comportamentais, cujos mecanismos de risco para as DIC não são conhecidos, em: problemas emocionais crônicos (ansiedade, depressão, hipocondriase, alteraçôes do sono, etc) e padrão de compor lamenio "lipo A" que se caracteriza por comportamentos como competividade, esforços intensos para realização, hostilidade facilmente provocável, sentimento de urgência para fazcr coisas e ser pontual, impaciência, gestos e fala rápidos, concentraçào em objetivos auto selecionados sem considerar outros aspectos do ambiente, inclusive as observaçðes e objetivos de outras pessoas, supervalorização da realização profissional ou vocacional em detrimento de outros aspectos da vida. Genericamente, as pessoas que têm comportamento opostos são definidos como um padrão comportamental "tipo B". 
Os estudos quanto às medidas para se modificar esses fatores ainda não são conclusivos. Sugere-se a orientação para exercícios regulares, relaxamento físico e mental e desenvolvimento de suporte familiar (ALLRED \& AYERS'). Além disso, as pessoas que se caracterizam por esses comportamentos ou estão nessas situaçoes devem receber orientaçðes quanto as possiveis intervençठes nos outros fatores de risco associados.

O importante é que qualquer esforço que altere esses comportamentos se torna válido por resultar, pelo menos, em uma vida mais agradável tanto para o próprio individuo quanto para sua familia.

\section{CONCLUSÃO}

Todas as medidas para modificação de fatores de risco das doenças isquêmicas do coração envolvem mudanças de comportamentos, eliminação e adoção de hábitos diferentes.

A análise da situação de cada paciente, cliente ou familia deve ser conjunta, isto é, o enfermeiro deve criar momentos para discutir com a pessoa ou familia sob seus cuidados quanto aos hábitos que tem e os possiveis riscos envolvidos afim de que se determine, conjuntamente, os comportamentos a serem modificados, estabelecendo-se metas a serem alcançadas (SHULTZ'r).

As responsabilidades do paciente, cliente ou familia, assim como as do enfermeiro devem ser definidas, e a avaliação da efetividade das medidas adotadas é fundamental para a realimentação do processo como um todo, devendo também ser feita pelo enfermeiro em conjunto com o paciente.

A modificação de comportamento não é um processo simples, totalmente conhecido, baseado somente na aquisição de algumas informaçōes, exigindo outras funçôes além da cognitiva. Por isso o trabalho a nivel de prevenção primária das DIC deve ser planejado e conduzido com a maior participação possivel da pessoa ou grupo a que se destina, considerando suas reais necessidades de saúde, crenças, valores e motivação.

Em função das dificuldades relativas às mudanças de comportamento tem se enfatizado a importância de que as crianças adquiram e mantenham um estilo de vida saudável e que reconheçam a saúde como um valor a ser preservado.

É possivel que a prevenção primária das DIC, iniciada na infância, tenha melhores resultados e pode partir de três aspectos relacionados ao estilo de vida: alimentação, atividade física e fumo (STRONG ${ }^{18}$ ).

CRUZ, D.A.L.M. da. Nursing aspects in primary prevention of isquemic heart disease. Rev. Esc. Enf. USP, Såo Paulo, 22(2):215-221, Aug. 1988.

This article presents the current informations on risk factors on the development of isquemic heart disease in order to support nurse's intervention in this field.

UNITERMS: Cardiology. Risk factors. Nursing care. 


\section{REFERE.NCIAS BIBLIOGRAFICAS}

1. ALLRED, C.A. \& AYtRS, C.R. The primary prevention of premature CVD: update. Occup. Health Nurs., Thorofare, 31(8):28-32, 1983.

2. ARONOW, W.S. \& KAPLAN, N.M. Smoking. In: KAPLAN, N.M. \& STAMLER, J. Prevention of coronary heart disease: practical management of the risk factors. Philadelphia, W.B. Saunders, 1983. p. $51-60$.

3. BRIODY, M.E. The role of the nurse in modification of cardiac risk factors. Nurs. Clin. North. Am., Philadelphia, 19(3):387-396, 1984.

4. DEL NERO, E. Fatores de risco para as doenças cardiovasculares. Ars. Curandi, São Paulo, 19(5):3-9, 1986.

5. GIANNINI, S.D. Fatores de risco coronariano. Arq. Bras. Cardiol. Sảo Paulo, $23($ ):345-352, 1970.

6. GOTTO, A.M. \& WITTELS, E.H. Diet, serum cholesterol, lipoproteins, and coronary heart disease. In: KAPLAN, N.M. \& STAMLER, J. Prevention of coronary heart disease: practical management of the risk factors. Philadelphia, W.B. Saunders, 1983. p. 33-50.

7. HEYDEN, S. Cardiologia preventiva. Mannheim, Boehringer Mannheim, 1983. 143p.

8. JENKINS, C.D. Psychosocial and behavioral factors. In: KAPLAN, N.M. \& STAMLER, J. Prevention of coronary heart disease: practical management of the risk factors. Philadelphia, W.B. Saunders, 1983. p. 98-112.

9. KANNEL, W.B. An overview of the risk factors for cardiovascular disease. In: KAPLAN, N.M. \& STAMLER, J. Prevention of coronary heart disease: practical management of the risk factors. Philadelphia, W.B. Saunders, 1983. p. 1-19.

10. KAPLAN, N.M. Diabetes and glucose intolerance. In: KAPLAN, N.M. \& STAMLER, J. Prevention of coronary heart disease: practical management of the risk factors. Philadelphia, W. B. Saunders, 1983. p. 113-119.

11. Hypertension. In: KAPI.AN, N.II. \& SIAMLER, J. Prevention of coronary heart disease: practical management of the risk facturs. Philadelphia, W.B. Saunders, 1983. p. 61-72.

12. KERN, L.S. \& GAWLINKI, A. Siage managing coronary artery disease. Nursing, Springhouse, 13(4):34-40, 1983.

13. LAURENTI, R. Epidemiologia das doenças cardiovasculares no Brasil. Arq. Bras. Cardiol., São Paulo, 38(4):243-248, 1982.

14. LEON, A.S. \& BLACKBURN, H. Physical inactivity. In: KAPL.AN, N.M. \& STAMLER, J. Prevention of coronary heart disease: practical management of the risk factors. Philadelphia, W.B. Saunders, 1983, p. 86-97.

15. LOLIO, C.A. \& LAURENTI, R. Mortalidade por doença isquêmica do coraçăo no Município de São Paulo: evolução de 1950-81 e mudanças recentes na tendência. Arq. Bras. Cardiol., Sảo Paulo, 46(3): $153 \cdot 156,1986$.

16. SHULTZ, C.M. Lifestyh assesment: a tool for practice. Nurs. Clin. North. Am., Philadelphia, $19(2): 271-81,1984$.

17. STAMLER, J. Coronary heart disease: doing the "right things" N. Fing. J. Med., Boston, 312(6): 1053-1055, 1985.

18. STRONG, W.B. Atheroscleroses: its pediatric roots. In: KAPLAN, N.M. \& STAMLER, J. Prevention of coronary heart disease: practical management of the risk factors. Philadelphia, W.B. Saunders, 1983, p.20-32.

19. TURPEINEM, O. Effect of cholesterol lowvering diet on mortality from CHD and their causes. Circulation, New York, 59(1):1-7, 1979.

20. WOLINSKY, H. Atheroscleroses. In: BEENSON, P.B. et alii. Cecil textbook of medicine. 15, ed Philadelphia, W.B. Saunders, 1979. p. 1218-1223. 